INPLASY

PROTOCOL

To cite: Yuan et al. The Efficacy and Safety of Onion Extract Gel On Scar Management: A Systematic Review and MetaAnalysis. Inplasy protocol 202080103. doi:

10.37766/inplasy2020.8.0103

Received: 25 August 2020

Published: 25 August 2020

Corresponding author: Jie Shen

doctorshenjie@sina.com

Author Affiliation:

Southwest Hospital, The Third Military Medical University (Army Medical University)

Support: Youth Talent Supported Program

Review Stage at time of this submission: Preliminary searches.

\section{The Efficacy and Safety of Onion Extract Gel On Scar Management: A Systematic Review and Meta-Analysis}

Yuan, X1; Shen, J2; Chen, L3; Wang, L4; Yan, Q5; Zhang, J6.

Review question / Objective: The purpose of this metaanalysis was to evaluate the efficacy and safety of OE gel on scar management and to provide reliable evidence for clinical application.

Condition being studied: Due to the reported effects of antiinflammatory, anti-microbial, anti-proliferative, and regenerative activities of onion extract (OE), its gel modality has been commercially available for treating, preventing, and reducing dermatologic scars and keloids in clinic many years. Several clinical trials found this gel was well tolerated and helpful for preventing pathological scarring and improving preexisting scars. Thus, it was recommended for clinical scar management by the International Advisory Panel on Scar Management in 2014. However, large-sized randomized controlled trials (RCTs) and the evidence-based data are still lacking.

INPLASY registration number: This protocol was registered with the International Platform of Registered Systematic Review and Meta-Analysis Protocols (INPLASY) on 25 August 2020 and was last updated on 25 August 2020 (registration number INPLASY202080103).

Conflicts of interest:

None.

\section{INTRODUCTION}

Review question / Objective: The purpose of this meta-analysis was to evaluate the efficacy and safety of OE gel on scar management and to provide reliable evidence for clinical application.
Condition being studied: Due to the reported effects of anti-inflammatory, antimicrobial, anti-proliferative, and regenerative activities of onion extract (OE), its gel modality has been commercially available for treating, preventing, and reducing dermatologic scars and keloids in clinic many years. 
Several clinical trials found this gel was well tolerated and helpful for preventing pathological scarring and improving preexisting scars. Thus, it was recommended for clinical scar management by the International Advisory Panel on Scar Management in 2014. However, large-sized randomized controlled trials (RCTs) and the evidencebased data are still lacking.

\section{METHODS}

Search strategy: Pubmed \#1 Allium cepa[TIAB] OR onion[TIAB] OR onions[TIAB] OR onion extract[TIAB] \#2 scar[TIAB] OR scars[TIAB] OR cicatrix[TIAB] OR cicatrice[TIAB] OR cicatrices[TIAB] OR keloid[TIAB] OR keloids[TIAB] OR cicatricle[TIAB] \#3 \#1 AND \#2 Medline \#1 TS=(Allium cepa) OR $\mathrm{TS}=$ (onion) OR TS=(onions) OR TS=(onion extract) \#2 TS=(scar) OR $\mathrm{TS}=$ (scars) OR $T S=$ (cicatrix) OR TS=(cicatrice) OR TS=(cicatrices) OR TS=(keloid) OR TS=(keloids) OR TS=(cicatricle) \#3 \#1 AND \#2 Embase and Cochrane Library \#1 Allium cepa:ti,ab,kw OR onion:ti,ab,kw OR onions:ti,ab,kw OR onion extract:ti,ab,kw \#2 scar:ti,ab,kw OR scars:ti,ab,kw OR cicatrix:ti,ab,kw OR cicatrice:ti,ab,kw OR cicatrices:ti,ab,kw OR keloid:ti,ab,kw OR keloids:ti,ab,kw OR cicatricle:ti,ab,kw \#3 \#1 AND \#2.

Participant or population: Patients with wounds or scars.

Intervention: Used topical gel with OE for scar management.

Comparator: Used no topical treatment, placebo gel, or other commonly used topical therapy for scar management.

Study designs to be included: Randomized controlled trials.

Eligibility criteria: Studies met the PICOS (patients, intervention, comparator, outcome, study design) criteria. (1) Patients: patients with wounds or scars. (2) Intervention: topical gel with OE. (3) Comparator: no topical treatment, placebo gel, or other commonly used topical therapy for scar management. (4) Reported at least one of the following outcomes: quantitative evaluation of the results by one scale, such as the Visual Analog Scale, Vancouver Scar Scale and Manchester Scar Scale, the width and/or volume of the scars, patient satisfaction, and adverse events. (5) Study design: RCT.

Information sources: Embase, PubMed, Medline and the Cochrane Library from inception to August 2020.

Main outcome(s): The width or volume of the scars, patient satisfaction, and adverse events.

Quality assessment / Risk of bias analysis: The quality of RCTs will be assessed according to the guidelines in the Cochrane Handbook for Systematic Reviews of Interventions to create a "risk of bias" table that included the following contents: details on methods of random sequence generation, allocation concealment, blinding, incomplete outcome data, selective outcome reporting, and other bias. The overall quality of each study will be evaluated as "low risk of bias", "high risk of bias", or "unclear risk of bias". Moreover, the RCTs will be quantitatively assessed by a modified Jadad 7-point scale and the Jadad score $\geq 4$ is considered to be of high-quality.

Strategy of data synthesis: Meta-analysis will be conducted by Review Manager software 5.3. Continuous outcomes will be assessed by mean difference (MD) with 95\% confidence intervals (Cls), while dichotomous outcomes will be evaluated by odds ratio (OR) with $95 \%$ Cls. Separate statistics will be combined by the inverse variance or Mantel-Haenszel method. P and 12 values will be used to assess heterogeneity among included studies. If $\mathbf{I 2}$ $<50 \%$ and $\mathrm{P}>0.1$, a fixed-effects model will be applied; otherwise, a randomeffects model will be applied.

Subgroup analysis: Subgroup analysis of patients with wounds or scars and the 
control group with or without other topical treatments will be performed. $P<0.05$ will be considered statistically significant.

Sensibility analysis: Sensitivity analysis will be conducted by omitting one study in turn.

Country(ies) involved: China.

Keywords: Onion extract; gel; scar; metaanalysis.

Contributions of each author:

Author 1 - Xi Yuan - Data curation, formal analysis, and writing original draft.

Author 2 - Jie Shen - Conceptualization, data curation, formal analysis, and writing review \& editing.

Author 3 - Li Chen - Methodology, data curation, and formal analysis.

Author 4 - Liang Wang - Software and writing original draft.

Author 5 - Qing Yan - Validation and writing original draft.

Author 6 - Jia Zhang - Validation and supervision. 